\title{
Coupling Strategies for the Synthesis of Peptide-Oligonucleotide Conjugates for Patterned Synthetic Biomineralization
}

\author{
Joshua D. Carter ${ }^{1}$ and Thomas H. LaBean ${ }^{1,2}$ \\ ${ }^{1}$ Departments of Computer Science and Chemistry, Duke University, Durham, NC 27708, USA \\ ${ }^{2}$ Department of Biomedical Engineering, Duke University Pratt School of Engineering, Durham, NC 27708, USA
}

Correspondence should be addressed to Thomas H. LaBean, thomas.labean@duke.edu

Received 17 May 2011; Accepted 25 June 2011

Academic Editor: F. C. Simmel

Copyright (c) 2011 J. D. Carter and T. H. LaBean. This is an open access article distributed under the Creative Commons Attribution License, which permits unrestricted use, distribution, and reproduction in any medium, provided the original work is properly cited.

\begin{abstract}
This work describes preparation strategies for peptide-oligonucleotide conjugates that combine the self-assembling behavior of DNA oligonucleotides with the molecular recognition capabilities of peptides. The syntheses include a solution-phase fragment coupling reaction and a solid-phase fragment coupling strategy where the oligonucleotide has been immobilized on DEAE Sepharose. The yield of four coupling reagents is evaluated, two reagents in water, EDC (1-ethyl-3-(3-dimethylaminopropyl) carbodiimide hydrochloride) and DMTMM (4-(4,6-dimethoxy[1,3,5]triazin-2-yl)-4-methyl-morpholinium chloride), and two in dimethylformamide (DMF), PyBOP ((Benzotriazol-1-yloxy) tripyrrolidinophosphonium hexafluorophosphate) and HBTU (Obenzotriazole- $N, N, N^{\prime}, N^{\prime}$-tetramethyluronium hexafluorophosphate), while the oligonucleotide fragment is either in solution or immobilized on DEAE. These coupling strategies rely on an unprotected $5^{\prime}$ amino linker on the oligonucleotide reacting with the peptide C-terminus. The peptide, selected from a combinatorial library for its gold-binding behavior, was 12 amino acids long with an N-terminus acetyl cap. Formation of the conjugates was confirmed by gel electrophoresis and mass spectrometry while molecular recognition functionality of the peptide portion was verified using atomic force microscopy. Solution-phase yields were superior to their solid-phase counterparts. EDC resulted in the highest yield for both solution-phase (95\%) and solid-phase strategies (24\%), while the DMF-based reagents, PyBOP and HBTU, resulted in low yields with reduced recovery. All recoverable conjugates demonstrated gold nanoparticle templating capability.
\end{abstract}

\section{Introduction}

Development of oligonucleotide-based therapies began thirty years ago with the demonstration of gene regulation using synthetic oligonucleotides $[1,2]$. Since that discovery, an increasing number of functional groups have been conjugated to oligonucleotides in efforts to enhance specific behaviors. Peptides, in particular, are an increasingly popular choice for conjugation with oligonucleotides because of their ability to aid in targeting specific biological functions while improving permeability through the cell membrane and stability against intracellular degradation. Peptideoligonucleotide conjugates (POCs) have been used to target mRNA, double-stranded DNA, and proteins, in antisense, triple-helix, and aptamer-based therapy, respectively [3-5]. Our group has recently explored another use of POCs by incorporating peptides used in artificial biomineralization [6-8] into self-assembling DNA nanostructures [9-11] for the demonstration of controlled biomineralization [12]. While the range of proposed applications for POCs continues to widen, scientists have relied on two principal strategies for joining the increasingly diverse peptide and oligonucleotide sequences together: in-line synthesis and fragment conjugation.

In-line synthesis typically involves the step-by-step synthesis of an oligomer covalently linked to a solid support and culminates with a linker through which the monomerby-monomer assembly of the second oligomer can take place. The use of a solid-support during in-line synthesis of POCs greatly reduces the complexity of purification and can also be useful for the production of POC libraries. Furthermore, immobilizing the oligomers on solid-phase 
eliminates the potential for differential solubility issues when attempting to synthesize a water-soluble oligonucleotide with a hydrophobic peptide component. However, difficulties in the production of POCs can arise due to the need for complex protecting group strategies to avoid damaging the first oligomer during synthesis of the second. This may lead to restrictions on sequence choices [13]. Moreover, each successive monomer addition results in a reduction of the overall yield making the production of long POCs difficult or impossible [14].

Fragment conjugation, in contrast to in-line synthesis, employs a strategy where the oligonucleotide and peptide are synthesized, deprotected, and purified separately, followed by a final step in which covalent linkage of the two pure fragments occurs. Although this strategy requires additional purification steps, it allows for the use of standard protecting group strategies in the production of the peptide and oligonucleotide fragments. The peptide does not need to be stable during oligonucleotide synthesis, and vice versa. Fragment conjugation also allows for the storage of the respective oligomer fragments for later use in multiple conjugate syntheses. Although fragment-coupling is rather straightforward, the relative solubilities of the oligomer fragments can cause problems. Oligonucleotides are water-soluble, while some peptides may be hydrophobic and require the use of organic solvents for coupling. One possible solution to this problem is the use of solid-phase fragment conjugation. In this strategy, the fragments are covalently linked while one oligomer is immobilized on solid-phase media.

Here, we describe both a solution-phase fragment coupling reaction and a nearly identical solid-phase fragment coupling where the oligonucleotide fragment has been temporarily immobilized on ion exchange resin via electrostatic interactions. Immobilizing the oligonucleotide fragment to a solid support allows us to consider only the solubility requirements of the peptide fragment when choosing a reaction solvent. The yields of both the solution-phase and solidsupported fragment coupling reactions were measured with two water-soluble coupling reagents, EDC and DMTMM, and two coupling reagents in dimethylformamide, PyBOP and HBTU. The coupling reaction relies on the formation of a peptide bond between an unprotected oligonucleotide with a $5^{\prime}$ amino linker and the C-terminus of a 12 amino acid gold-binding peptide with an acetyl-capped $\mathrm{N}$-terminus [15]. Characterization of the synthetic strategies using polyacrylamide gel electrophoresis and mass spectrometry will be presented, the effect of reaction solvent and coupling reagent will be discussed, and the choice of solution-phase or solid-phase media will be evaluated. Finally, incorporation of the purified POC into a self-assembling DNA lattice allows for atomic force microscope evaluation of the gold-binding capability of the free-peptide end.

\section{Methods and Materials}

2.1. Purification of Oligonucleotide. /5AmMC6/TTG TGA AGT TTT TCG ATC CTA GCA CCT CTG GAG TTT TTC TTG CC. Synthetic oligonucleotide with a $5^{\prime}$-Amino Modifier C6 functional group was purchased from Integrated
DNA Technologies (Coralville, IA) and separated from truncation products by polyacrylamide gel electrophoresis. Denaturing polyacrylamide gel electrophoresis (PAGE) was carried out on a $160 \times 180 \times 1.5 \mathrm{~mm}$ gel containing $10 \%$ acrylamide and $8.3 \mathrm{M}$ urea in TBE buffer $(90 \mathrm{mM}$ Tris, $90 \mathrm{mM}$ boric acid, and $2 \mathrm{mM}$ ethylenediaminetetraacetate, $\mathrm{pH} 8$ ). The gel loading buffer contained $90 \%$ formamide and $0.1 \%$ bromophenol blue. After electrophoresis $(300 \mathrm{~V}$ for 45 minutes), the portion of the gel containing the desired DNA was excised, diced, and shaken overnight at $4^{\circ} \mathrm{C}$ in $500 \mu \mathrm{L}$ $0.5 \mathrm{M}$ ammonium acetate, $10 \mathrm{mM}$ magnesium acetate, and $2 \mathrm{mM}$ ethylenediaminetetraacetate. The supernatant was removed and added to a centrifuge tube with $1 \mathrm{~mL}$ of $100 \%$ $(\mathrm{v} / \mathrm{v})$ ethanol and stored at $-20^{\circ} \mathrm{C}$ overnight. The mixture was centrifuged for 30 minutes at $4^{\circ} \mathrm{C}$ and $16,000 \mathrm{~g}$ and the supernatant was discarded. The purified pellet of DNA was dissolved in pure water and then desalted using a Pierce spin column (\#69705) containing Sephadex G-25 Medium (Amersham Biosciences). The concentration was determined by ultraviolet absorption at $260 \mathrm{~nm}$ wavelength.

2.2. Peptide Synthesis and Purification. The gold-binding peptide [15] (Ac-WALRRSIRRQSY-OH) was synthesized on Wang resin preloaded with Fmoc-L-Tyrosine (Novabiochem) at $0.1 \mathrm{mmoL}$ scale using a Protein Technologies PS3 automated peptide synthesizer. The coupling of standard Fmoc (9-fluorenylmethoxy-carbonyl)-protected amino acids (Chem-Impex) was achieved with HBTU (O-benzotriazole$N, N, N^{\prime}, N^{\prime}$-tetramethyluronium hexafluorophosphate; Novabiochem) in the presence of $N$-methylmorpholine (NMM) in $N, N^{\prime}$-dimethylformamide (DMF) for 20-minute cycles. Fmoc deprotection was achieved using $20 \%$ piperidine in DMF $(2 \times 5$ minutes $)$. The $\mathrm{N}$-terminus of the peptide was acetylated with acetic anhydride and NMM. Side-chain deprotection and peptide cleavage from the resin were achieved by treating the resin-bound peptide with $5 \mathrm{~mL}$ of $100 \%$ trifluoroacetic acid (TFA) for 2 hours under $\mathrm{N}_{2}$. After evaporation of TFA under $\mathrm{N}_{2}$, the peptide was washed three times with cold diethyl ether, air-dried, and purified by semipreparative reverse-phase HPLC on a YMC C18 column with a linear 40-minute gradient from 3 to $70 \%$ acetonitrile in water with $0.1 \%$ TFA. The mass of the peptide was confirmed using an Agilent ESI-MS.

2.3. Reaction Solvents in Peptide-Oligonucleotide Synthesis. Fragment-coupling reactions using PyBOP ((Benzotriazol1-yloxy) tripyrrolidinophosphonium hexafluorophosphate; Aldrich) and HBTU were run completely in DMF using DNA immobilized on DEAE Sepharose media (DFF-100, Sigma). Reactions with water-soluble coupling reagents EDC (1ethyl-3-(3-dimethylaminopropyl) carbodiimide hydrochloride) and DMTMM (4-(4,6-dimethoxy[1,3,5] triazin-2-yl)4-methyl-morpholinium chloride) were completed using both Sepharose media and in solution-phase. DMTMMbased coupling reactions were run in $200 \mathrm{mM}$ MOPS buffer pH 7.0 (200 mM (3[N-morpholino]propanesulfonic acid, $20 \mathrm{mM}$ sodium acetate, $10 \mathrm{mM}$ EDTA) while EDC-based reactions were run in water. 
TABLE 1: Reaction yields for POC formation in the listed conditions.

\begin{tabular}{lcccccc}
\hline Reaction & Coupling reagent & Solvent & Supporting media & Temperature & Reaction time & Yield $(\%)$ \\
\hline 1 & HBTU & DMF & Sepharose & room temperature & $16 \mathrm{~h}$ & 0 \\
2 & PyBOP & DMF & Sepharose & room temperature & $16 \mathrm{~h}$ & 4 \\
3 & DMTMM & Water & Sepharose & room temperature & $16 \mathrm{~h}$ & 15 \\
4 & EDC & Water & Sepharose & room temperature & $16 \mathrm{~h}$ & 24 \\
5 & DMTMM & Water & Sepharose & $50^{\circ} \mathrm{C}$ & $16 \mathrm{~h}$ & 15 \\
6 & DMTMM & Water & Sepharose & room temperature & $2 \mathrm{~h}$ & $2 \mathrm{~h}$ \\
7 & PyBOP & DMF & Sepharose & room temperature & 15 \\
8 & DMTMM & Water & Solution & room temperature & $16 \mathrm{~h}$ & 50 \\
9 & EDC & Water & Solution & room temperature & $16 \mathrm{~h}$ & 55 \\
10 & DMTMM & Water & Solution & $50^{\circ} \mathrm{C}$ & 55 \\
\hline
\end{tabular}

2.4. General Procedure for Coupling Reactions. All reactions were run in a constant volume in the solvent preferred by the coupling reagent. For DMTMM-coupled reactions, 450 pmoles of DNA was combined with peptide ( 270 nmoles, 600 eq.) and DMTMM (4.5 $\mu$ moles, 10,000 eq.). EDC-based coupling reactions followed the same procedure as above using EDC ( $4.5 \mu$ moles, 10,000 eq $)$ and N-hydroxy succinimide ( 450 nmoles, 1000 eq) in place of DMTMM. PyBOP and HBTU-based coupling reactions were accomplished in DMF with PyBOP or HBTU ( $4.5 \mu$ moles, 10,000 eq.) and diisopropylethylamine (9.0 $\mu$ moles, 20,000 eq.).

2.5. Workup for Solution-Phase Coupling Reactions. The peptide-oligonucleotide conjugates were isolated from the completed solution-phase coupling reactions via ethanol precipitation. In this case, $1 \mathrm{~mL}$ of $100 \%(\mathrm{v} / \mathrm{v})$ ethanol and $50 \mu \mathrm{L}$ of $3 \mathrm{M}$ sodium acetate ( $150 \mu$ moles) were added and allowed to sit overnight at $-20^{\circ} \mathrm{C}$. The precipitated conjugate was centrifuged for 30 minutes at $4^{\circ} \mathrm{C}$ and $16,000 \mathrm{~g}$ and the supernatant was discarded. The pellet was dried in a vacuum centrifuge for 2 hours before resuspension in $30 \mu \mathrm{L}$ water and analysis by denaturing PAGE (300 V for 45 minutes).

2.6. General Procedure for Solid-Phase Coupling Reactions. The procedure for immobilization of DNA on Sepharose was adapted from Halpin et al. [16]. Briefly, $250 \mu \mathrm{L}$ of Sepharose slurry was added to a Pierce spin column, promptly washed with three column volumes of DNAloading buffer $(10 \mathrm{mM}$ Acetic Acid and $0.005 \%$ Triton X100 ), and briefly centrifuged (one minute at $3,000 \mathrm{~g}$ ). To bind the DNA on the Sepharose, 450 pmoles DNA $(250 \mu \mathrm{L}$ loading buffer) was incubated with the Sepharose resin for ten minutes and centrifuged for one minute at 3,000 g. The resin was then washed with one column volume of the appropriate reaction solvent (DMF, buffer, or water) before addition of the solution containing coupling reagent and peptide to the immobilized DNA. The resulting mixture was allowed to react for the duration listed in Table 1. After the reaction, the exhausted reagents were removed using a centrifuge ( 1 minute at $3,000 \mathrm{~g}$ ) and the column was washed with one column volume of DNA loading buffer. The peptide-oligonucleotide conjugate was eluted with $2 \times$ $200 \mu \mathrm{L}$ elution buffer $(1.5 \mathrm{M} \mathrm{NaCl}$ and $50 \mathrm{mM}$ Tris- $\mathrm{HCl}$
$\mathrm{pH} 8.0$ ) and the combined fractions were added to $1 \mathrm{~mL}$ of $100 \%$ (v/v) ethanol and allowed to sit overnight at $-20^{\circ} \mathrm{C}$. The precipitated DNA was centrifuged for 30 minutes at $4^{\circ} \mathrm{C}$ and $16,000 \mathrm{~g}$ and the supernatant was discarded. The pellet was dried in a vacuum centrifuge for 2 hours before resuspension in $30 \mu \mathrm{L}$ water and analysis using denaturing PAGE (300 V for 45 minutes).

2.7. Denaturing PAGE Analysis of the Reaction Products. To determine the yield of a coupling reaction, $15 \mu \mathrm{L}$ of the DNA recovered from the reaction was added to a $10 \%$ denaturing polyacrylamide gel and run at $300 \mathrm{~V}$ for 45 minutes. The gel containing the contents of the peptide-oligonucleotide conjugate reaction was stained with ethidium bromide and imaged with a UV transilluminator. Reaction yields were determined by comparing fluorescent signals from unreacted oligonucleotide (faster moving band) to coupled peptide-oligonucleotide conjugate (slower moving band) using the UV transilluminator software (AlphaImager HP from AlphaInnotech).

2.8. MALDI Analysis of Peptide-Oligonucleotide Conjugates. MALDI-TOF mass spectrometry analysis was used to characterize the oligonucleotide starting material and peptideoligonucleotide reaction product. The analysis was performed using an Applied Biosystems DE-Pro Maldi-MS in the Mass Spectrometry Facility in the Chemistry Department at Duke University. To prepare the samples, the products were recovered from the polyacrylamide gels (vide supra) and dissolved in pure water to a concentration of $30 \mu \mathrm{M}$. $10 \mu \mathrm{L}$ volumes of the recovered gel products were then stripped of cations using Ziptips (SCX, Millipore) and added to a mixture of $9 \mu \mathrm{L}$ of $50 \mathrm{mg} / \mathrm{mL} 3$-hydroxypicolinic acid and $1 \mu \mathrm{L} 50 \mathrm{mg} / \mathrm{mL}$ diammonium citrate. The mass spectrometer was run in negative-ion mode and spectra were collected through the summing of 50 laser pulses.

2.9. DNA Strands. The self-assembling DNA lattice used in these experiments is described elsewhere [12].

2.10. DNA Nanostructure Formation. For DNA lattice formation, 18 individual PAGE-purified DNA oligos and the 
peptide-oligo conjugate (POC) were mixed together stoichiometrically at $1.0 \mu \mathrm{M}$ in $1 \times \mathrm{TAE} / \mathrm{Mg}^{2+}$ buffer $(40 \mathrm{mM}$ Tris- $\mathrm{HCl}$ ( $\mathrm{pH} 8.0$ ), $20 \mathrm{mM}$ acetic acid, $2 \mathrm{mM}$ EDTA, and $12.5 \mathrm{mM}$ magnesium acetate) and slowly cooled from 95 to $20^{\circ} \mathrm{C}$ over a period of 16 hours. For AFM imaging, $3 \mu \mathrm{L}$ of sample was spotted on freshly cleaved mica for 3 minutes. A $30 \mu \mathrm{L}$ portion of $1 \times \mathrm{TAE} / \mathrm{Mg}^{2+}$ buffer was then placed onto the mica and another $30 \mu \mathrm{L}$ of $1 \times \mathrm{TAE} / \mathrm{Mg}^{2+}$ buffer was placed onto the AFM tip (for a total of $60 \mu \mathrm{L}$ ). AFM images were obtained on a Digital Instruments Nanoscope IIIa with a multimode head by tapping mode under buffer using NP-S tips (Veeco Inc.).

2.11. Gold Nanoparticle Preparation. Gold nanoparticles (AuNP, $5 \mathrm{~nm}$ ) were purchased from Ted Pella (product no. 15702-20). To prevent nanoparticle aggregation in the high salt environment preferred by the DNA lattice, nanoparticles were pretreated with Tween 20 (Sigma Aldrich product no. P1379). $1 \mathrm{~mL}$ of stock AuNP solution was mixed with the appropriate amount of Tween 20 stabilizing agent to achieve a final Tween concentration of $0.2 \%(\mathrm{w} / \mathrm{v})$. After addition of the Tween 20, the AuNP solutions were mixed overnight at room temperature. After incubation, the AuNP particles were concentrated by 6 hours of centrifugation at $16000 \mathrm{~g}$. The resulting AuNP pellet was then isolated using a pipet and resuspended in $10 \mu \mathrm{L}$ of supernatant. The concentrations of the AuNP were determined using the AuNP absorbance at $520 \mathrm{~nm}\left(\varepsilon 520 \mathrm{~nm}\right.$ for $5 \mathrm{~nm}$ AuNP $\left.=1 \times 10^{7} \quad \mathrm{M}^{-1} \mathrm{~cm}^{-1}\right)$ [17].

2.12. AFM Imaging of AuNP-Labeled DNA Lattice. AuNPlabeled DNA lattice was prepared by adding $6 \mu \mathrm{L}$ annealed lattice solution to concentrated AuNP in $1 \times \mathrm{TAE} / \mathrm{Mg}^{2+}$ buffer. four equivalents of AuNP to one equivalent of POC were used in all cases. All samples combining DNA with AuNP were allowed to mix in solution for 10 minutes prior to deposition of $3 \mu \mathrm{L}$ of the mixture on mica, followed by a 3 minute wait before the addition of $60 \mu \mathrm{L}$ of buffer for tapping mode AFM analysis. AFM images were obtained on a Digital Instruments Nanoscope IIIa with a multimode head by tapping mode under buffer using NP-S tips (Veeco Inc.).

\section{Results and Discussion}

3.1. Peptide Synthesis and Purification. A recently published peptide sequence (Ac-WALRRSIRRQSY-OH) was selected to serve as the test molecule for making peptide-oligonucleotide conjugates [15]. The peptide, known for its ability to bind gold surfaces, was synthesized according to the procedure detailed in the methods and materials. Briefly, Fmoc coupling protocols were used to add amino acid residues to Wang resin preloaded with Fmoc-L-Tyrosine. The Nterminus of the peptide was acetylated to prevent the primary amine from participating in the coupling reaction with DNA. The final product was purified by semipreparative HPLC and analysis showed a purity greater than 95\%. ESI-MS measurements of the final product gave an $\mathrm{m} / \mathrm{z}$ of 1634.2

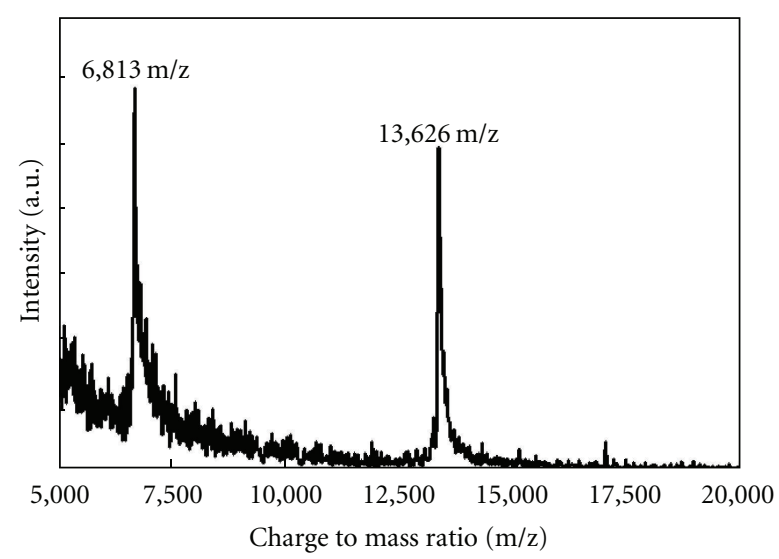

(a)

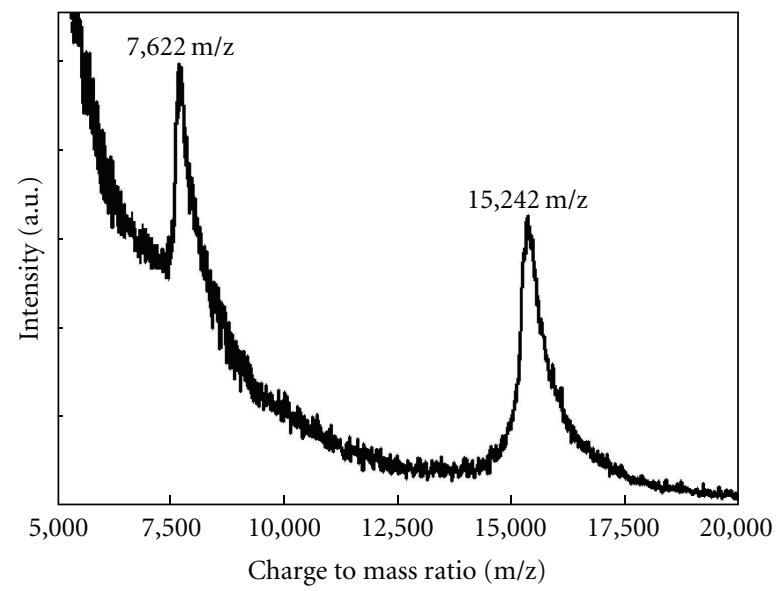

(b)

Figure 1: MALDI-TOF analysis of pure oligonucleotide starting material (a) and peptide-oligonucleotide conjugate (b).

$\left(\mathrm{M}+\mathrm{H}^{+}\right)$which compared well with the calculated mass of $1633.9 \mathrm{~g} / \mathrm{mol}$.

3.2. Oligonucleotide Purification. The oligonucleotide sequence (TTG TGA AGT TTT TCG ATC CTA GCA CCT CTG GAG TTT TTC TTG CC) used in the test couplings was selected from a DNA nanostructure previously constructed in our lab [9]. Prior to coupling, the commercially synthesized oligonucleotide, containing a primary amine on its $5^{\prime}$ end, was dissolved in pure water and purified using PAGE. After purification, the recovered DNA pellet was dissolved in pure water and desalted using size exclusion chromatography to produce the purified oligonucleotide in $30 \%$ yield. Incomplete removal of the salts remaining from DNA synthesis and purification was found to have an adverse effect on the DNA affinity for the Sepharose reaction media (data not shown).

3.3. MALDI Analysis of Peptide-Oligonucleotide Conjugates. Figure 1 shows the spectra obtained for pure oligonucleotide starting material and the peptide-oligonucleotide conjugate. As expected for the starting material, the measured mass 


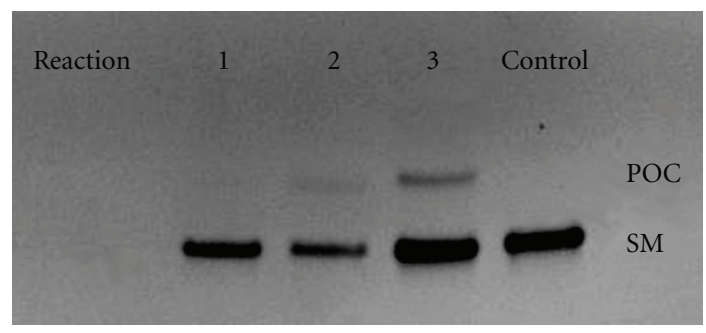

Figure 2: PAGE analysis of reactions 1-3 from Table 1. The coupling reagents used in lanes 1, 2, and 3 are HBTU, PyBOP, and DMTMM, respectively. As demonstrated by the control lane, starting material (SM) is below the slower-moving peptideoligonucleotide conjugate (POC).

of $13,626 \mathrm{~m} / \mathrm{z}$ corresponds well with the calculated theoretical mass of $13,630.9 \mathrm{~g} / \mathrm{mol}$. Similarly for the peptide oligonucleotide conjugate, the observed mass of $15,242 \mathrm{~m} / \mathrm{z}$ corresponds well with the calculated theoretical mass of $15,246.8 \mathrm{~g} / \mathrm{mol}$.

\subsection{Effects of Reaction Variables on Coupling Efficiency.} The coupling reagents and reaction variables tested for this report are detailed in Table 1. Conditions varied for this study include coupling reagent and solvent, reaction temperature, and reaction duration. Table 1 also delineates reactions that occurred on the Sepharose media and those that took place in solution. Reactions were evaluated based on the yield of peptide-oligonucleotide conjugate obtained for each coupling reagent. The reaction yield was determined by integrating the fluorescent signal from ethidium bromide-stained PAGE and comparing between unreacted oligonucleotide and newly formed (and slower moving) peptide-oligonucleotide conjugate. The reaction yield, or the amount of product obtained for each reaction, should be distinguished from the total recovery of DNA, the sum of product and unreacted oligonucleotide.

Reactions 1-4 listed in Table 1 compare the coupling efficiency of four coupling reagents at room temperature over 16 hours while the DNA is confined to the Sepharose surface. Under these conditions, EDC was the most effective coupling reagent and produced the highest reaction yield of $24 \%$. At the other extreme, PyBOP and HBTU produced little or no product. PyBOP and HBTU also resulted in a reduced total recovery of the DNA from the Sepharose. This effect, demonstrated in Figure 2 (lanes 1 and 2) as a relatively weak fluorescent signal from the total DNA present in each lane, is in agreement with the findings of other researchers [16]. Aqueous washes of the Sepharose columns were used after DNA loading when coupling with either EDC or DMTMM and DMF washes were used after DNA loading in cases where PyBOP or HBTU was to be the coupling reagent. Although enhanced DNA solubility in the DMF wash solvent might have been suspected for the poor recovery of DNA from these latter reactions, PAGE analysis of DMF wash fractions collected from the Sepharose column demonstrated the absence of prematurely eluted DNA. This indicates irreversible binding between the DNA and the Sepharose for PyBOP and HBTU-based reactions in DMF, but this could not be directly measured.

To test the effect of temperature on yield, reaction 3 (DMTMM, Sepharose) was repeated at a higher temperature (Reaction 5). As shown in Table 1, increasing the temperature to $50^{\circ} \mathrm{C}$ resulted in no increase in the yield for the DMTMM coupling reagent on Sepharose over 16 hours. Reaction 3 was repeated again on a shorter time scale (Reaction 6, Table 1) producing little effect on the yield indicating that the formation of peptide-oligonucleotide product is essentially complete within two-hour reaction time. Previous research into formation rates of peptide-oligoconjugates has demonstrated nearly complete reactions in as few as twenty minutes [18]. Although the quick completion of the reaction could be due to decay of the DMTMM through demethylation or hydrolysis, other groups have found the decay in this short time frame to be insignificant $[19,20]$. The effect of decreasing reaction duration on coupling yield was repeated with PyBOP (Reaction 7), and as in the case of DMTMM, very little change in yield was observed. Reactions 8 and 9 are the solution-phase equivalent to the solidphase reactions 3 and 4, respectively. As the data illustrate, dissolving the DNA in solution rather than binding it to Sepharose led to a dramatic increase in the reaction yield. One possible explanation for this observation is that the DNA conformation resulting from the electrostatic interactions between the Sepharose and the DNA could be adversely affecting the progress of the reaction. These interactions may prevent the amine group from reacting with the carboxylic acid of the peptide [21]. In the case of solution-phase coupling, DMTMM produced 50\% product while EDC went nearly to completion (95\%). The effect of temperature on DMTMM coupling was again tested in reaction 10, and in this case, a small improvement in the yield was observed.

\subsection{Gold-Binding Functionality of the POCs after Incorpo-} ration into DNA Lattice. POC preparation reactions that produced yields of at least $15 \%$ allowed for the purification and isolation of POC material (Table 1). To test the gold-binding capability of these POC materials, they were incorporated into the modified DNA lattice prior to addition of $5 \mathrm{~nm}$ AuNP and analysis with AFM. Figure 3 contains typical AFM images of both unmodified DNA lattice and DNA lattice incorporating POC after addition of $5 \mathrm{~nm}$ AuNP while Figure 4 shows the corresponding line profiles for both samples. AFM analysis of DNA lattices incorporating purified POC species isolated from the various preparation reactions listed in Table 1 showed no discernable difference in the areal density of AuNP reached with identical reaction times and AuNP concentrations. The steady goldbinding functionality of the purified POCs synthesized by the various means leads to two possible conclusions, either the peptide consistently formed a covalent linkage to the oligonucleotide as designed through the $5^{\prime}$ amino linker on the oligonucleotide and the $\mathrm{C}$-terminus of the peptide, or the gold-binding functionality of the peptide is not constrained by the location of the covalent linkage between oligonucleotide and peptide. Due to the uncertainty surrounding the gold-binding mechanism of the peptide either of these 


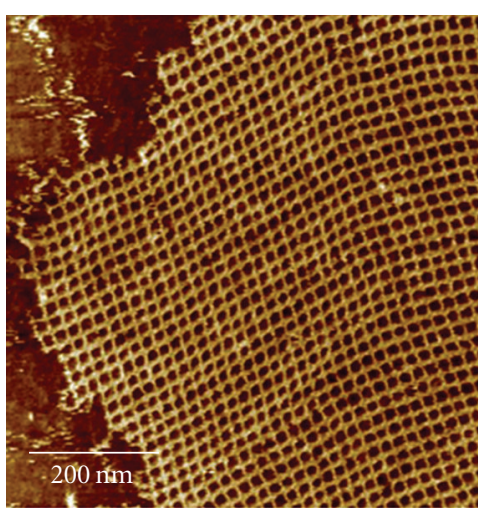

(a)

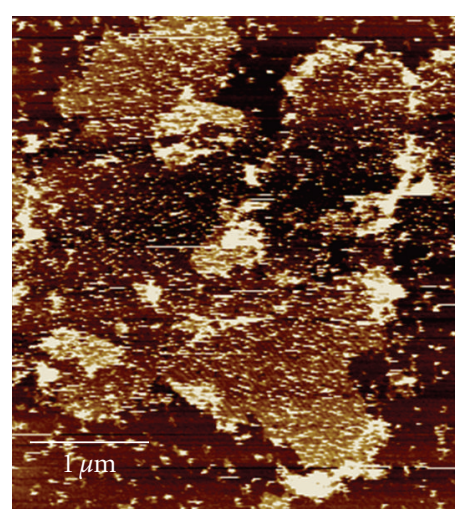

(b)

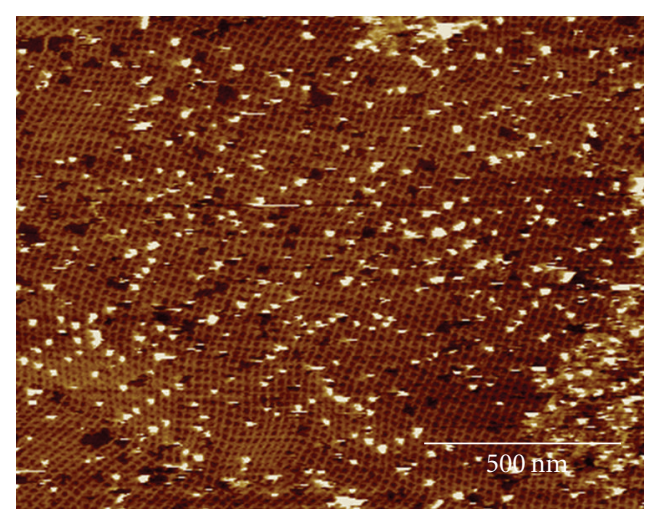

(c)

FIgURe 3: AFM images of a DNA lattice (a) and a DNA lattice incorporating POC isolated from reaction 3 after addition of $5 \mathrm{~nm}$ gold nanoparticles (b) and (c).

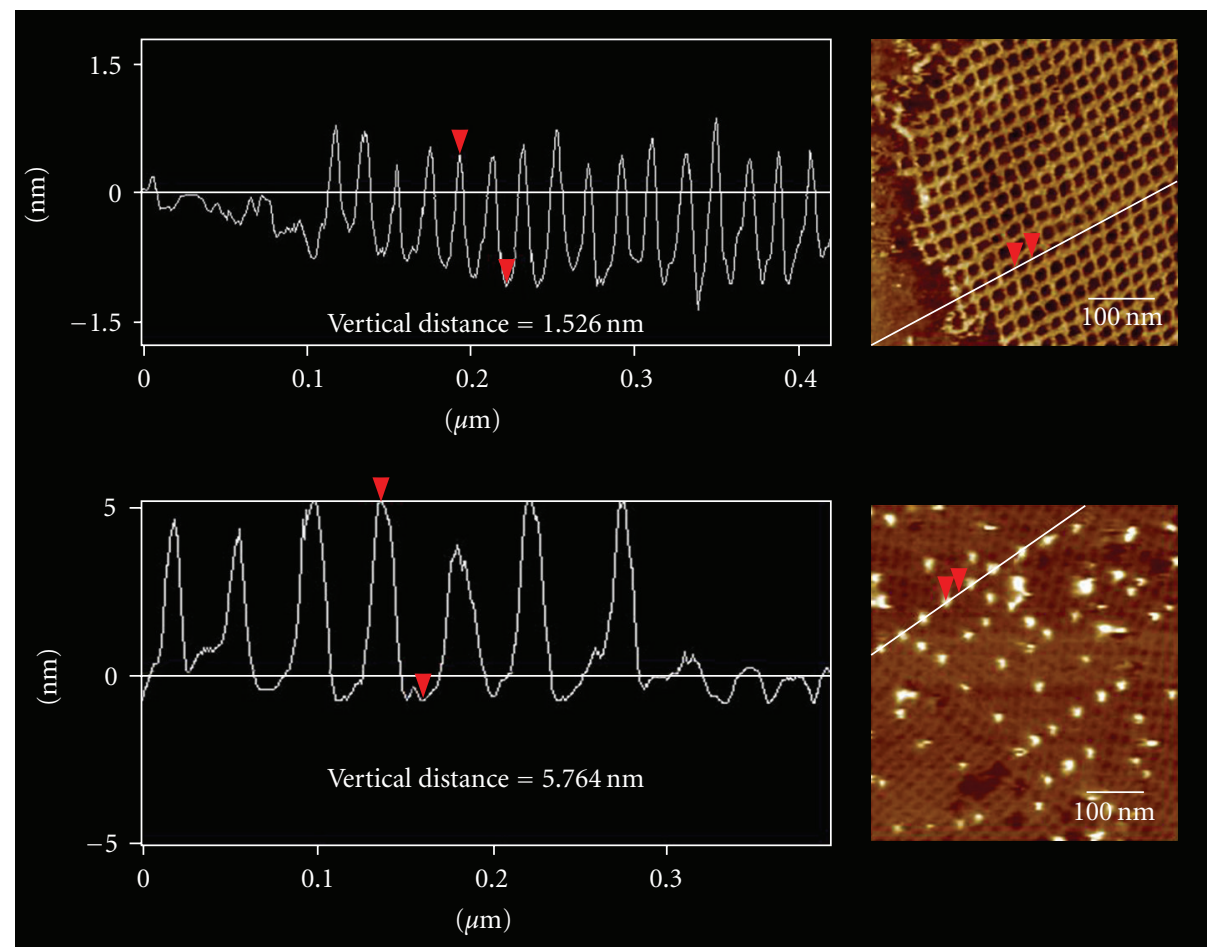

Figure 4: AFM profile scans of a DNA lattice (top) and a DNA lattice incorporating POC isolated from reaction 3 after addition of $5 \mathrm{~nm}$ gold nanoparticles (bottom).

scenarios remains plausible. Although higher areal densities have been achieved by using AuNP labeled with singlestranded DNA complementary to strands incorporated into DNA lattice [22], the simultaneous organization of multiple inorganic species favors an approach with greater selectivity, such as those exhibited by proteins in biological chemistry. Furthermore, experimental evidence indicated an inverse correlation between the number of AuNP equivalents added and the binding time required to reach near-saturating conditions. This suggests that low AuNP areal densities can be improved with increased reaction time and additional AuNP equivalents.

\section{Conclusion}

The work presented herein describes multiple strategies for the preparation of POCs that combine the capabilities of both self-assembling DNA oligonucleotides with the molecular-recognition capabilities of peptides. Four coupling reagents have been evaluated for their ability to form peptide oligonucleotide conjugates while the oligonucleotide is either in solution-phase or restricted to a solid-support. The molecular recognition capability of the peptide ends of the POC products was further evaluated using $5 \mathrm{~nm}$ AuNP after their incorporation into a self-assembling DNA 
lattice. The various purified POCs, when incorporated into DNA lattices and allowed to mix with AuNP for equivalent durations, resulted in equivalent areal densities of AuNP when visualized using AFM. In the preparation of the POCs, EDC consistently produced the highest yield while watersoluble coupling reagents as a whole produced markedly higher yields compared to their hydrophobic counterparts. Solution-phase coupling yields were superior to their solidphase alternative. DMF-based reactions resulted in reduced recovery of coupled product from the solid-phase resin. Furthermore, when choosing between DMF and water for the formation of peptide-oligonucleotide conjugates, the superior yields of EDC and DMTMM demonstrated here indicate water to be the superior solvent for coupling. However, researchers attempting to form peptide-oligonucleotide conjugates in the future may choose solvents other than water in order to balance starting material solubility with reaction yield. It is worth noting that although the results in this report were obtained using a water-soluble gold-binding peptide, the coupling yield of hydrophobic peptides targeting other species may be different under otherwise identical conditions.

In the characterization of peptide functionality after anchoring to a DNA oligonucleotide, all reactions from which viable yields of POC could be isolated demonstrated consistent nanoparticle templating capability. Although not tested for this report, there are studies concluding that the length of the amine linker may also affect the yield of the coupling reactions $[18,23]$. This effect could provide researchers with additional means of optimizing the yield of peptide-oligoconjugate reactions. Another factor not tested for this report was the scalability of the coupling reactions. The manufacturer-published exchange capacity for DEAE Sepharose gel of $110-160 \mu \mathrm{eq} / \mathrm{mL}$ means that the saturation point for the DNA used in this report is at least three orders of magnitude higher, allowing for the potential use of larger reaction scales.

\section{Acknowledgments}

The authors thank Katherine Franz for the use of the peptide synthesizer, Kurt Gothelf and Niels Vinther Voigt for experimental advice, and George Dubay for assistance with the MALDI measurements. The authors acknowledge funding for this project from NIH training Grant T32-EB001630 (JDC) and NSF Grants CCF-SGER-0650083, DMR-BMAT0706397, and CCF-EMT-0829749.

\section{References}

[1] M. L. Stephenson and P. C. Zamecnik, "Inhibition of Rous sarcoma viral RNA translation by a specific oligodeoxyribonucleotide," Proceedings of the National Academy of Sciences of the United States of America, vol. 75, no. 1, pp. 285-288, 1978.

[2] P. C. Zamecnik and M. L. Stephenson, "Inhibition of Rous sarcoma virus replication and cell transformation by a specific oligodeoxynucleotide," Proceedings of the National Academy of Sciences of the United States of America, vol. 75, no. 1, pp. 280284, 1978.
[3] J. Oehlke, G. Wallukat, Y. Wolf et al., "Enhancement of intracellular concentration and biological activity of PNA after conjugation with a cell-penetrating synthetic model peptide," European Journal of Biochemistry, vol. 271, no. 14, pp. 30433049, 2004.

[4] D. S. Wilson and J. W. Szostak, "In vitro selection of functional nucleic acids," Annual Review of Biochemistry, vol. 68, pp. 611647, 1999.

[5] O. A. Sedelnikova, V. N. Karamychev, I. G. Panyutin, and R. D. Neumann, "Sequence-specific gene cleavage in intact mammalian cells by ${ }^{125}$ I-labeled triplex-forming oligonucleotides conjugated with nuclear localization signal peptide," Antisense and Nucleic Acid Drug Development, vol. 12, no. 1, pp. 43-49, 2002.

[6] M. Sarikaya, C. Tamerler, A. K. Y. Jen, K. Schulten, and F. Baneyx, "Molecular biomimetics: nanotechnology through biology," Nature Materials, vol. 2, no. 9, pp. 577-585, 2003.

[7] J. N. Cha, G. D. Stucky, D. E. Morse, and T. J. Deming, "Biomimetic synthesis of ordered silica structures mediated by block copolypeptides," Nature, vol. 403, no. 6767, pp. 289-292, 2000.

[8] E. C. Samano, M. Pilo-Pais, S. Goldberg, B. N. Vogen, G. Finkelstein, and T. H. LaBean, "Self-assembling DNA templates for programmed artificial biomineralization," Soft Matter, vol. 7, no. 7, pp. 3240-3245, 2011.

[9] H. Yan, S. H. Park, G. Finkelstein, J. H. Reif, and T. H. LaBean, "DNA-templated self-assembly of protein arrays and highly conductive nanowires," Science, vol. 301, no. 5641, pp. 18821884, 2003.

[10] H. Li, J. D. Carter, and T. H. LaBean, "Nanofabrication by DNA self-assembly," Materials Today, vol. 12, no. 5, pp. 20-28, 2009.

[11] N. C. Seeman, "DNA in a material world," Nature, vol. 421, no. 6921, pp. 427-431, 2003.

[12] J. D. Carter and T. H. Labean, "Organization of inorganic nanomaterials via programmable DNA self-assembly and peptide molecular recognition," ACS Nano, vol. 5, no. 3, pp. 2200-2205, 2011.

[13] C. H. Tung and S. Stein, "Preparation and applications of peptide-oligonucleotide conjugates," Bioconjugate Chemistry, vol. 11, no. 5, pp. 605-618, 2000.

[14] N. Venkatesan and B. H. Kim, "Peptide conjugates of oligonucleotides: synthesis and applications," Chemical Reviews, vol. 106, no. 9, pp. 3712-3761, 2006.

[15] M. Hnilova, E. E. Oren, U. O. S. Seker et al., "Effect of molecular conformations on the adsorption behavior of goldbinding peptides," Langmuir, vol. 24, no. 21, pp. 12440-12445, 2008.

[16] D. R. Halpin, J. A. Lee, S. J. Wrenn, and P. B. Harbury, "DNA display III. Solid-phase organic synthesis on unprotected DNA," PLoS Biology, vol. 2, no. 7, p. E175, 2004.

[17] T. A. Taton, "Preparation of gold nanoparticle-DNA conjugates," Current Protocols in Nucleic Acid Chemistry, vol. 12, no. 2, p. 11, 2002.

[18] S. M. Viladkar, "Guanine rich oligonucleotide-amino acid/ peptide conjugates: preparation and characterization," Tetrahedron, vol. 58, no. 3, pp. 495-502, 2002.

[19] K. Hojo, M. Maeda, N. Tanakamaru, K. Mochida, and K. Kawasaki, "Solid phase peptide synthesis in water VI: evaluation of water-soluble coupling reagents for solid phase peptide synthesis in aqueous media," Protein and Peptide Letters, vol. 13, no. 2, pp. 189-192, 2006. 
[20] M. Kunishima, C. Kawachi, J. Morita, K. Terao, F. Iwasaki, and S. Tani, "4-(4,6-Dimethoxy-1,3,5-triazin-2-yl)-4-methylmorpholinium chloride: an efficient condensing agent leading to the formation of amides and esters," Tetrahedron, vol. 55, no. 46, pp. 13159-13170, 1999.

[21] D. C. Harris, X. Chu, and J. Jayawickramarajah, "DNA-small molecule chimera with responsive protein-binding ability," Journal of the American Chemical Society, vol. 130, no. 45, pp. 14950-14951, 2008.

[22] J. Zhang, Y. Liu, Y. Ke, and H. Yan, "Periodic square-like gold nanoparticle arrays templated by self-assembled 2D DNA nanogrids on a surface," Nano Letters, vol. 6, no. 2, pp. 248251, 2006.

[23] S. Peyrottes, B. Mestre, F. Burlina, and M. J. Gait, "The synthesis of peptide-oligonucleotide conjugates by a fragment coupling approach," Tetrahedron, vol. 54, no. 41, pp. 1251312522, 1998. 

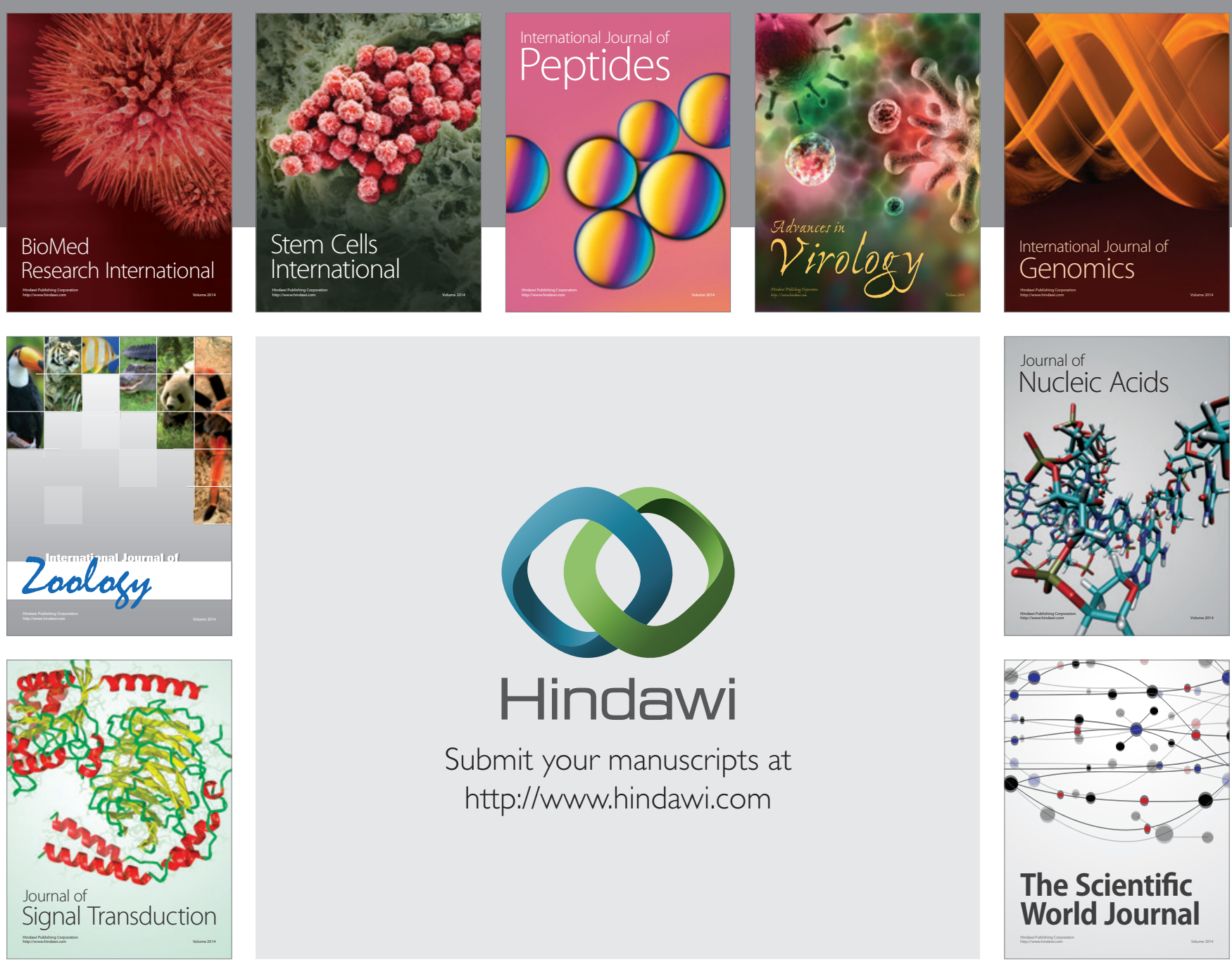

Submit your manuscripts at

http://www.hindawi.com
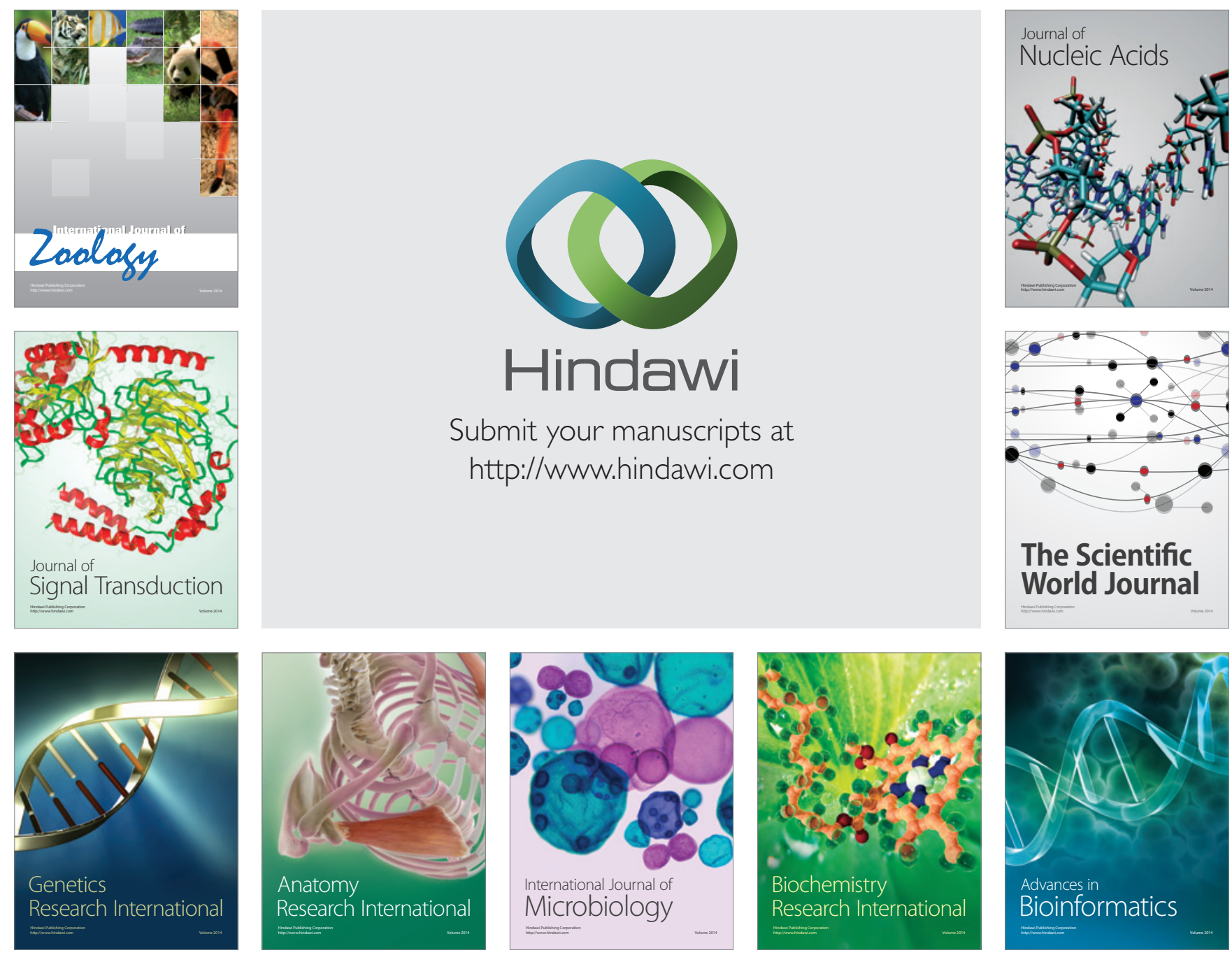

The Scientific World Journal
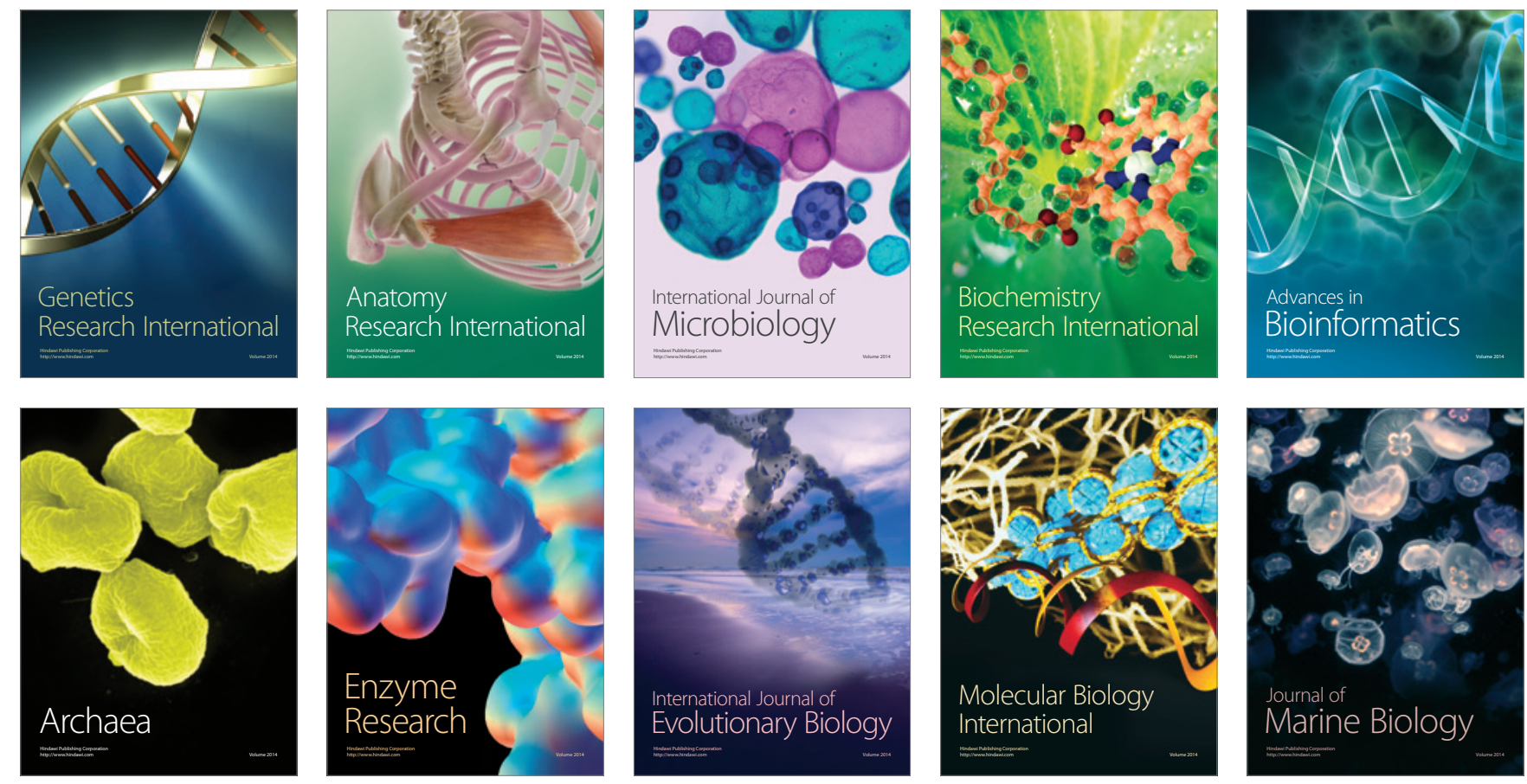